\title{
Direct lineage reprogramming for brain repair: Breakthroughs and challenges
}

Rory Vignoles $^{1 *}$, Célia Lentini ${ }^{1 *}$, Marie d'Orange ${ }^{1}$ and Christophe Heinrich ${ }^{1 \#}$

${ }^{1}$ University of Lyon, University Claude Bernard Lyon 1, Inserm, Stem Cell and Brain Research Institute U1208, 69500 Bron, France

${ }^{*}$ Equal contribution

${ }^{\#}$ Correspondence: Christophe.heinrich@inserm.fr (C. Heinrich)

Phone: +33472913482

\section{Keywords}

Brain repair; Direct reprogramming; Cell-fate conversion; Glial cells

\begin{abstract}
Injury to the human CNS is devastating because our adult mammalian brain lacks intrinsic regenerative capacity to replace lost neurons and induce functional recovery. An emerging approach towards brain repair is to instruct fate conversion of brain-resident non-neuronal cells into induced neurons (iNs) by direct lineage reprogramming. Considerable progress has been made in converting various source cell types of mouse and human origin into clinically-relevant iNs. Recent achievements using transcriptomics and epigenetics have already shed light on molecular mechanisms underpinning neuronal reprogramming, while the potential capability of iNs in promoting functional recovery in pathological contexts has started to be evaluated. Although future challenges need to be overcome before clinical translation, lineage reprogramming holds promise for effective cell-replacement therapy in regenerative medicine.
\end{abstract}




\section{Direct lineage reprogramming: A new avenue towards brain repair}

Injury to the human central nervous system (CNS) is devastating because our adult mammalian brain has little, if any, intrinsic regenerative capacity to replace lost neurons and induce functional recovery. Hence acute CNS injury and chronic neurodegenerative diseases are associated with irreversible loss of neurons, ultimately leading to permanent functional deficits and neurological disability. The main goal of regenerative medicine for brain repair is to replace lost neurons in order to restore lost functions. Classical cell-replacement approaches, such as transplantation of stem cells and their derivatives into the injured brain, still face major obstacles preventing translation into clinically-effective therapies [1]. Therefore, it is crucial to open doors to alternative therapeutic strategies to ultimately target these unmet medical needs.

An innovative and exciting approach towards achieving replacement of damaged neurons is to induce directly in vivo the fate conversion of non-neuronal cells residing within the injured brain into neurons, by a process referred to as direct lineage reprogramming. Lineage reprogramming consists in assigning a new cellular identity to terminally differentiated somatic cells. Pioneering work by several labs successfully converted in vitro various source cell types of mouse and human origin into clinically-relevant induced neurons (iNs) by forced expression of selected transcription factors (TFs) [2-14], including the proneural pioneer TFs Ascl1 or Neurog2, or by exposure to small molecules $[8,9,14,15]$. Importantly, several groups now achieved in vivo lineage reprogramming within the murine brain by instructing endogenous non-neuronal cells to generate functional iNs $[9,16-25]$. Highlighting the dynamic of this fast-moving field, an increasing number of studies has already shed light on the molecular mechanisms underpinning direct neuronal reprogramming by studying the transcriptomic and epigenetic modifications imposed by reprogramming factors $[7,10,26-28]$. In the meantime, other labs have embarked on evaluating the potential capability of iNs in promoting functional recovery in a pathological context [9], which is a fundamental question for brain repair.

Here, we review the main achievements in lineage reprogramming of various brain-resident cell types into distinct types/subtypes of iNs that have been generated in diverse brain regions using various reprogramming routes. Furthermore we discuss the future challenges arising on the 
road to successful use of direct lineage reprogramming as a viable therapeutic approach for brain repair.

\section{Direct reprogramming of astroglia into neurons}

An inspired strategy aiming at reprogramming glial cells into clinically-relevant iNs has emerged over the last decades with the underlying rationale to recruit glia as an endogenous cellular source for brain repair. Efforts have largely sought to take advantage of reactive gliosis (see Glossary) accompanying most neurological disorders associated with neuronal death $[29,30]$.

\section{Cortical astrocytes}

When looking for best-suited cells as endogenous sources for neuronal reprogramming, astrocytes and more particularly reactive astrocytes imposed themselves as appealing candidates being located at the injury site and resuming proliferative capability [30,31]. Notably, astrocytes also share a common embryological origin with neurons and derive from the common ancestor radial glial cells [29] that may ease their reprogramming towards a neuronal identity. Pioneering this approach, the Götz lab first showed that non-neurogenic astroglia isolated in vitro from the postnatal mouse cerebral cortex (Figure 1A, Table 1) could be instructed to generate iNs by retrovirally-mediated expression of a single neurogenic TF such as Pax6 [2] or proneural TFs Neurog2 or Ascl1 [3]. Next, the study by Heinrich et al. (2010) showed that strong and persistent expression of neurogenic fate determinants could instruct postnatal astroglia to mature into fully functional, synapse-forming neurons [4,5]. Importantly, the neurotransmitter fate choice of astroglia-derived iNs could be controlled by selective expression of distinct TFs: While the dorsal telencephalic fate determinant Neurog2 directed cortical astroglia to generate glutamatergic neurons, the ventral telencephalic determinant DIx2 induced a GABAergic identity, albeit with lower efficiency [4,5,32]. Employing genetic fate-mapping, Heinrich et al. (2010) could unambiguously show direct conversion of fate-mapped cortical astroglia into iNs [4]. Following studies revealed that postnatal cortical astrocytes could also be converted into glutamatergic iNs upon forced expression of NeuroD1 [22] or NeuroD4 combined with Insm1 [7], or into midbrain dopaminergic iNs by combined expression of Ascl1, Lmx1b and Nurr1 [33]. 
Based on successful generation of astroglia-derived iNs in vitro, the quest for inducing neurogenesis in vivo in the injured brain has rapidly become a major goal. Antagonizing Olig2 function or inducing Pax6 expression in reactive cells proliferating in response to stab-wound injury in the adult mouse cerebral cortex (Figure 1B) promoted acquisition of neuronal traits [16]. The Nakafuku lab showed that retrovirally-driven expression of Neurog2, but not Ascl1, in non-neuronal cells proliferating after stab-wound injury in the adult rat neocortex induced Doublecortin (DCX)positive cells, an effect that was robustly enhanced with addition of growth factors [19]. At that time the identity of the cells serving as source for iNs remained unclear, and only a limited fraction of iNs matured further expressing $\mathrm{NeuN}$ and glutamate at later stages [19]. Of note, an ischemia insult strongly enhanced both iN production and maturation compared to stab injury. The Chen lab then reported that reactive astrocytes in the cortex of stab-injured mice or Alzheimer's disease mouse model could be efficiently reprogrammed by NeuroD1 into functional iNs [22]. Interestingly, co-expression of Neurog2 and Bcl-2 in glial cells following cortical stab injury also induced efficient conversion into NeuN-positive iNs and additional treatment with vitamin $\mathrm{E}$ or a vitamin $\mathrm{D}$ receptor ligand further improved maturation into Ctip2-positive iNs [8].

\section{Striatal astrocytes}

In the adult mouse striatum, Parmar and colleagues showed that combinatorial expression of Ascl1, Brn2a and Myt1l in astrocytes induced their conversion towards a neuronal identity (Figure 1E) [18]. In parallel, the Nakafuku lab observed that retrovirally-mediated Neurog2 expression in cells proliferating following stab-wound injury in the rat striatum (Figure 1F) elicited appearance of DCX/NeuN-positive neurons, most being immunoreactive for GABA and Isl1, reminiscent of differentiating striatal projection neurons [19]. Remarkably, lentivirally-targeted expression of Sox2 in adult mouse quiescent striatal astrocytes was sufficient to convert them into Ascl1-positive neural progenitors which subsequently generated DCX-positive, proliferative neuroblasts [20,23]. Maturation of Sox2-induced neuroblasts into functional NeuN/calretinin-positive neurons required, however, additional treatments with BDNF (brain-derived neurotrophic factor) and the BMP (bone morphogenetic protein) antagonist noggin, or a histone deacetylase inhibitor $[20,23]$. Of note, Ascl1 itself did not evoke neurogenesis [23] even when combined with growth factors [19], and 
maintained Sox2 expression might be detrimental for survival and expansion of induced neuroblasts [20]. Interestingly, transitioning through intermediate progenitors endowed with proliferative ability before adoption of a mature neuron fate may have the added benefit to expand the pool of iNs.

From a translational perspective, generating dopaminergic iNs represents an attractive strategy toward cell-replacement therapy in Parkinson's disease. Using a mouse model of Parkinson's disease, the Arenas lab showed that targeted expression of NeuroD1, Ascl1, Lmx1a, and miR-218 to striatal astrocytes induced appearance of dopamine neurons expressing midbrain markers, as tyrosine hydroxylase $(\mathrm{TH})$ and dopamine transporter [9]. Surprisingly, although few iNs exhibited electrophysiological features of midbrain dopamine neurons, motor function deficits were restored in lesioned mice treated with reprogramming factors, suggesting iN ability to correct deficits in dopamine transmission [9]. However, ectopic striatal TH/Nurr1-positive neurons were also reported to appear spontaneously in the dopamine-denervated striatum in the same mouse model, thus challenging the above interpretation that $\mathrm{TH}$-positive cells arise from reprogramming [25].

Together, there is now a large body of evidence for conversion of cortical and striatal astrocytes into clinically-relevant iNs in vitro and in the injured brain in vivo.

\section{Direct reprogramming of NG2 glia into neurons}

\section{Cortical NG2 glia}

In the quest for identifying brain-residents suitable for reprogramming, NG2 glia also appear attractive candidates being endowed with interesting features from a translational perspective. NG2 glia continue dividing in the adult brain, although very slowly, and exhibit rapid and massive increase in proliferation following CNS injury [29]. Furthermore, NG2 cell loss triggers rapid migration and proliferation of adjacent cells to restore their density [34]. This therefore suggests that NG2 glia proliferating in the injured area could be targeted by viral vectors inducing their neuronal conversion, and that converted NG2 cells would be replaced through homeostatic control of density, thus avoiding depletion of local NG2 glia population. Employing a genetic fate-mapping strategy, Heinrich et al. (2014) showed that NG2 glia proliferating in the stab-injured cortex of adult 
mice could be reprogrammed into DCX-positive iNs by combined expression of Ascl1 and Sox2 (Figure 1D) [21]. Intriguingly, Sox2 alone was also sufficient to induce neuronal conversion of cortical NG2 glia, despite its known involvement in self-renewal regulation of neural stem cells (NSC) [35], but in line with Sox2-mediated conversion of striatal astrocytes into neuroblasts [20]. While generated in a context of severe injury, NG2 glia-derived iNs acquired the capacity to fire action potentials and received synaptic inputs from endogenous GABAergic neurons, indicating some degree of functional integration [21]. In addition, NeuroD1 expression in reactive NG2 cells in the stab-injured cortex instructed their conversion toward neurogenesis, and let to the conversion of postnatal cortical NG2 glia into glutamatergic or GABAergic iNs in vitro [22].

\section{Striatal NG2 glia}

Targeting expression of Ascl1/Lmx1a/Nurr1 to striatal NG2 cells induced generation of iNs firing action potentials and receiving synaptic inputs from local striatal projection neurons (Figure $1 \mathrm{H}$ ) $[24,25]$. Surprisingly, however, while these reprogramming factors have been shown to convert glia in vitro towards a dopaminergic phenotype [33], Ascl1/Lmx1a/Nurr1-iNs in the striatum essentially expressed GAD65/67 or vGlut1 in absence of TH expression, irrespective of their NG2 glia or astrocyte origin [24]. Most iNs displayed firing properties reminiscent of fast-spiking striatal interneurons and expressed interneuron-related genes including parvalbumin and somatostatin [25]. Intriguingly, iNs derived from striatal NG2 glia seem to be strongly committed to an interneuron identity, independently of the proneural genes (Ascl1, Neurog2, NeuroD1) and dopaminergic fate determinants employed (Lmx1a, Nurr1, FoxA2, En1), even in a context of dopamine denervation [25].

In summary, similarly to astrocytes, numerous studies have demonstrated efficient NG2 glia-to-neuron conversion upon expression of various TFs.

\section{Impact of brain environment and source cell type on direct reprogramming}

Distinct reprogramming outcomes in terms of efficiency and neuronal specification were obtained while using the same TFs in different source cell types in vitro and in vivo, as illustrated for Ascl1 inducing neuronal conversion of postnatal cortical astrocytes in vitro [3,4], but failing in doing so in 
adult striatal astrocytes [23] and cortical NG2 glia in vivo [21]. This raises the crucial question of how neuronal identity and subtype specification are assigned during direct lineage conversion. Reprogramming mechanisms appear to be controlled in a source cell type-dependent manner $[36,37]$, but the local microenvironment in which conversion has to occur in vivo [38] and injurymediated signaling [39] seem to also have a strong impact on reprogramming efficiency and subtype specification (Figure 3, Key Figure).

\section{Impact of distinct brain areas on in vivo reprogramming}

While Sox2 induced neurogenesis in striatal astrocytes [20], it failed doing so in cortical glia in absence of prior injury [21]. In addition, while Neurog2 stimulated generation of mature iNs in the stab-injured striatum, this effect was way more restricted in the neocortex [19]. These results therefore suggest that the striatum is a more permissive structure supporting neurogenesis. This is supported by the observation that DCX/NeuN-positive cells can be spontaneously observed following stab-injury in the adult striatum unlike in the neocortex [19]. Reinforcing this notion, stroke injury in the striatum [40] or excitotoxic lesion in a model of Huntington's disease [41] were also shown to elicit a neurogenic program in striatal astrocytes in absence of reprogramming factors, which resulted from Notch1 signaling downregulation in the case of stroke [40].

Interestingly, the molecular phenotype of iNs engineered by similar manipulations was also different depending on the brain area where iNs were generated. For instance, Neurog2-iNs exhibited features resembling glutamatergic neurons in the injured neocortex, while they were reminiscent of differentiating striatal projection neurons in the injured striatum $[8,19]$. Furthermore, the fact that both striatal NG2 glia and astrocytes were converted into inhibitory neurons regardless of the TFs employed [23,25], may suggest preferential reprogramming towards GABAergic identity in the striatum. It is therefore tempting to speculate that extrinsic signaling cues of the neighboring environment may superimpose onto converting cells, a particular neuronal subtype identity beyond the sole control of reprogramming genes. 


\section{Impact of source cell types}

Besides the microenvironment, distinct source cell types exhibit specific reprogramming competences, likely due to specific chromatin landscape, epigenetic signature, and accessibility to various target genes required for neuronal conversion (Figure 3), thereby explaining various reprogramming efficiency and differentiation outcomes $[7,10,28]$. Such reprogramming competence may be linked to the developmental origin of source cell types. Along this line, cortical astrocytes sharing a common embryological origin with neurons and deriving from radial glial cells [29], may be endowed with intrinsic reprogramming competence towards glutamatergic identity $[4,5,7,8,15,19,38,42]$, whereas their conversion into GABAergic lineages is limited $[4,5,42]$. Similarly, during embryonic development, NG2 glia derive from neural progenitor cells in the ganglionic eminence that also generate GABAergic interneurons [43], suggesting that sharing a developmental origin with interneurons could ease reprogramming toward this subtype, potentially explaining conversion of striatal NG2 cells into interneurons regardless of fate determinants, even when TFs known to control dopaminergic fate were employed $[24,25]$.

Adding an additional layer of complexity, a given source cell type, as astrocytes, may exhibit distinct reprogramming competence depending on CNS regions of origin. Cortical astrocytes exhibited higher susceptibility to Neurog2- or Ascl1-reprogramming in vitro compared to spinal cord astrocytes, an effect mediated at least in part by increased levels of Notch1 signaling in less competent astrocytes [44]. In addition, cerebellar astrocyte-derived iNs adopted mostly a GABAergic phenotype following Neurog2-reprogramming [38], contrary to cortical astrocytes entering the glutamatergic lineage $[4,7,8,38]$.

Together, while TFs orchestrate neuronal conversion, efficiency and selective subtype specification appear strongly influenced by both intrinsic cell reprogramming competence and extrinsic environmental cues. To bring neuronal reprogramming closer to a clinical strategy in cell based-regenerative medicine, it will be required to identify such instructive factors to better control specificity of neuronal identity. 


\section{Direct reprogramming of human brain-resident cells into neurons}

From a translational perspective, a key issue was whether the human brain also contains cells amenable to neuronal reprogramming. Berninger and colleagues showed that pericytes isolated in vitro from cortical tissues surgically resected from adult human patients could be instructed by Ascl1/Sox2 co-expression to generate human iNs (hiNs) (Figure 2B) that were able to fire action potentials and received synaptic contacts $[6,10]$. While Ascl1/Sox2 synergize to mostly induce a forebrain GABAergic identity with emergence of distinct interneuron subtypes as evidenced by cholecystokinin or somatostatin expression, a subset of Ascl1/Sox2-iNs expressed Neurog2, NeuroD1, and NeuroD4 and were committed to a glutamatergic lineage [10]. Shedding light on pericytes as new candidates for reprogramming, these data opened new avenues towards use of endogenous cells to replace lost neurons in the adult human brain. Subsequently, human astrocytes isolated from fetal cortex could be converted into hiNs after grafting in rats, providing proof-of-principle for in vivo neuronal conversion of human astrocytes (Figure 2A) [18]. Additional studies showed that human fetal astrocytes could also be converted in vitro into hiNs by NeuroD1 [22], or NeuroD4 alone or in combination with Insm1, Prox1 or Sox11 (Figure 2D) [7].

Generating glia-derived dopamine hiNs represents a promising strategy towards cellreplacement therapy in Parkinson's disease. Immortalized human fetal astrocytes were reprogrammed into dopaminergic hiNs by a combination of NeuroD1/Ascl1/Lmx1a/miR-218 together with sequential treatment with small molecules promoting chromatin remodeling and activating TGF- $\boldsymbol{\beta}$, sonic hedgehog, and Wnt signaling pathways (Figure 2C) [9]. However, despite acquisition of midbrain regional identity, only few hiNs generated action potential [9]. Interestingly, human primary embryonic astrocytes could be converted by the same combination of TFs and small molecules into hiNs exhibiting electrophysiological features typical of midbrain dopamine neurons [9].

These studies demonstrated that human non-neuronal cells can be efficiently reprogrammed into hiNs by TF expression and/or treatment with small molecules (Box1). 


\section{Deciphering molecular mechanisms underpinning direct neuronal conversion of brain-resident cells}

Reprogramming factors impose on the differentiated source cell new transcriptional networks driving a new molecular program able to reassign the novel cellular identity (Figure 3 ). While astrocytes in culture and reactive astrocytes in vivo share the common feature of being proliferative, proliferation per se is not a sine qua non condition for successful astrocyte-to-neuron reprogramming $[4,8,38]$. Indeed, video-tracking revealed that most astrocytes could be converted into iNs in absence of cell division $[4,8,38]$, in contrast to reprogramming towards induced pluripotent stem cells [45]. Nonetheless, their proliferative status prior to reprogramming likely impacts the chromatin landscape of specific genes implicated in fate conversion. Along this line, cortical astroglia at later stages of differentiation become less amenable to Neurog2-mediated conversion due to reduced Neurog2 accessibility to its binding sites in the promoters of its direct downstream targets including NeuroD1 and NeuroD4 [7]. Reprogramming could be rescued by providing those astroglia with combined expression of NeuroD4 and Insm1, Prox1, or Sox11 [7]. Noteworthy, impaired Neurog2 binding to target loci resulted from binding of REST, known to repress neuronal genes [46], to the NeuroD4 locus, and also from chromatin remodeling as evidenced by enrichment in the heterochromatin-associated histone mark H4K20me3 [7]. Consistently, REST deletion in astroglia drastically enhanced Neurog2-reprogramming efficiency but also induced neurogenesis in absence of Neurog2 supply [7]. Thus, REST and repressive marks at essential neuronal genes are critical obstacles to be overcome for astrocyte-to-neuron conversion.

Matsuda and colleagues recently shed light on epigenetic remodeling associated with transcriptional regulation during neuronal reprogramming using microglia, the third cell type to partake in reactive gliosis [28]. Targeted NeuroD1 expression in microglia in vitro and in the adult mouse striatum induced conversion into functional iNs able to integrate into existing networks (Figure 1C,G) [28]. Characterization of transcriptional changes highlighted a two-step role of NeuroD1 first initiating the neuronal program prior to suppressing microglial genes [28]. NeuroD1 initially binds to closed chromatin with bivalent modifications including active (H3K4me3) and repressive (H3K27me3) marks to induce neuronal genes. These chromatin regions are 
subsequently resolved to monovalent active state by NeuroD1 itself, altering the epigenome by inducing genes involved in epigenetic modifications and chromatin remodeling. Thereafter, NeuroD1 induces transcriptional repressors that silence microglial/immune-cell specific genes, while NeuroD1 reprograms the microglial epigenetic landscape around promoter and enhancer regions to suppress microglial identity [28].

To unravel cell-dependent reprogramming routes leading to distinct neuronal phenotypes but also mechanisms responsible for reprogramming failures, single-cell transcriptome profiling has proved to be of great value $[10,26,27]$. Dissecting reprogramming trajectories will also answer the fundamental question whether direct lineage reprogramming corresponds to a direct fateswitch between two terminally differentiated cell states or rather rely on transition through intermediate states including reversion to a developmentally immature state [47]. Using single-cell transcriptome analysis, the Berninger lab identified two populations of adult human brain pericytes exhibiting distinct competence to Ascl1/Sox2-induced neuronal reprogramming [10]. Intriguingly, successful Ascl1/Sox2-mediated pericyte-to-neuron conversion included transient activation of a NSC-like intermediate state before bifurcation towards GABAergic or glutamatergic lineages [10]. Inhibition of the signaling pathways identified during this NSC-like state, such as BMP, Activin/Nodal, TGF- $\beta$, or Notch signaling increased reprogramming efficiency, while blocking BMP signaling further promoted iN maturation. These data thus support the notion that Ascl1/Sox2 activate hierarchical developmental gene regulatory networks $[10,47]$. It remains to be determined whether transition through NSC-like state could be related to Sox2, known to be essential for pluripotency [48] and NSC maintenance [49], rather than a mandatory step on reprogramming routes, as NeuroD1-driven microglia-to-neuron conversion occurs without NSC intermediate state $[28]$.

Another important aspect to consider for successful reprogramming is the metabolic state of the source and desired cell types as well as the related metabolic shift that cells have to properly negotiate during fate conversion (Figure 3). While astroglia rather use anaerobic glycolysis, neurons employ oxidative metabolism [50]. Thus transition towards neuronal oxidative metabolism could be accompanied by disturbed redox-homeostasis and reactive-oxygen species production impairing reprogramming. Importantly, Götz and colleagues demonstrated that metabolic shift is 
indeed a prerequisite for cell-fate transition during neuronal reprogramming, which causes oxidative stress representing a major obstacle for successful conversion [8]. Inhibiting oxidative stress-dependent ferroptosis promoted cell survival and Ascl1-mediated astrocyte-to-neuron conversion efficiency [8]. Furthermore, protecting astrocytes undergoing Ascl1- or Neurog2reprogramming from lipid peroxidation with the antioxidant vitamin $E$, Forskolin, vitamin $D$ receptor or Bcl-2 drastically improved iN generation [8]. Furthermore, neuroinflammation following injury also stimulates oxidative stress [51] that could further affect fate conversion and iN survival. Accordingly, glia-to-neuron conversion was more effective in the uninjured striatum $[18,20,24,25]$ or following mild injury in the cerebral cortex [22] than after severe invasive cortical injury [16,21]. Further highlighting the benefit of antioxidants, Bcl-2/Neurog2 co-expression in cortical glial cells induced neuronal reprogramming with high efficiency following stab-injury in vivo, and additional treatment with Calcitriol or vitamin E potently improved iN maturation into NeuN/Ctip2-positive neurons [8].

Together, recent achievements using transcriptomics and epigenetics, and metabolic studies shed new light on molecular mechanisms underlying neuronal reprogramming.

\section{Concluding remarks}

Reprogramming brain residents into iNs (Box2) has emerged as a promising cell-replacement strategy allowing use of endogenous patient-specific cells for brain repair. Before direct lineage reprogramming could reach clinical applications, fundamental questions need to be tackled (Outstanding Questions and Clinician's Corner). One challenge is to achieve in vivo reprogramming of residents into functional iNs that acquire the same molecular identity and phenotype as lost endogenous neurons within a given brain area and pathological condition. So far numerous in vivo studies mostly relied on immunostaining to characterize iN subtype specification, and electrophysiology was predominantly used to ascertain neuronal identity based on basic features. There is therefore a critical need to implement extensive characterization with electrophysiology of the specific iN phenotypes. Whether iNs generated in a given brain region may be restricted towards the generation of a specific neuronal subtype remains to be elucidated. The ability to generate diverse neuronal phenotypes may be a crucial step towards restoring a 
damaged or imbalanced neuronal network. Transcriptomics and epigenetics provided new insights into molecular mechanisms underpinning neuronal reprogramming, allowing identifying specific cell populations with distinct reprogramming competence and dissecting trajectories towards neuronal subtype specification. Further unraveling molecular programs driven by specific sets of TFs in diverse source cell types will reveal whether reprogramming events are largely shared by various donor cells or rather cell type-specific. It remains to be determined how epigenetic barriers in source cell types influence reprogramming and subtype specification outcomes. Furthermore, it will be essential to determine whether reprogramming pathways identified in vitro also hold true for in vivo lineage conversion, considering the impact of brain environment and injury-mediated signaling on reprogramming within the diseased brain.

Efficient reprogramming will require that iNs functionally integrate within pathological networks. To be clinically relevant, iNs must not only act as passive recipients of synaptic inputs from endogenous neurons, but also have to send axons and signals to their endogenous counterparts, a prerequisite for functional neurogenesis and repair. Along this line, several studies revealed that iNs received synaptic contacts, yet the identity of presynaptic partners remains to be disclosed in most cases. Whether iNs form a functional presynaptic output, and what is more, specifically target the expected neurons according to an effective repair strategy has yet to be demonstrated.

A major challenge will be to generate iNs that are endowed with the ability to restore lost synaptic transmission and modulate with beneficial effects the activity of pathological networks in which they incorporate, in order to ultimately provide functional rescue and correct neurological deficits. With respect to our current state of expertise, it will likely be difficult to reconstitute diffuse projection systems and complex circuits including distinct types/subtypes of lost neurons, such as in Alzheimer's disease or stroke involving widespread damage. Instead, restoring neurons of a single phenotype, and what is more in a single location, appear more realistic at this early stage. Along this line, besides conversion of striatal glia into dopaminergic iNs in animal models of Parkinson's disease, selective regeneration of relay neurons after spinal cord injury [52] or striatal medium spiny neurons in Huntington's disease could be envisaged for instance to provide solid proof-of-evidence for functional recovery provided by iNs. 
In conclusion, in vivo lineage reprogramming holds promise as a cell-replacement strategy to restore lost neurons. Nonetheless, moving from bench to bedside will require tackling the challenges arising on the road to its successful use as a viable therapeutic approach for CNS repair.

\section{Acknowledgements}

Due to the large amount of literature on direct lineage reprogramming, we wish to apologize to all authors whose important work could not be mentioned in the present review due to space restrictions. C.H. is supported by Agence Nationale de la Recherche (ANR, ReprogramEpi, ANR14-CE13-0001), LabEx CORTEX (ANR-11-LABX-0042) of Lyon University within the program "Investissements d'Avenir" (ANR-11-IDEX-0007) operated by ANR, Fédération pour la Recherche sur le Cerveau (FRC), Fondation Française pour la Recherche sur l'Epilepsie (FFRE), and Citizens United for Research in Epilepsy (CURE, Award ID : 262178). R.V. is supported by a PhD fellowship from Région Rhône-Alpes (ARC2, Qualité de Vie et Vieillissement, subvention \#16005489-01). C.L. is supported by a PhD fellowship from Ligue Française contre l'Epilepsie (LFCE). All authors approved the final version of the manuscript.

\section{References}

1 Lindvall, O. (2012) Why is it taking so long to develop clinically competitive stem cell therapies for CNS disorders? Cell stem cell 10, 660-662

2 Heins, N. et al. (2002) Glial cells generate neurons: the role of the transcription factor Pax6. Nature neuroscience 5, 308-315

3 Berninger, B. et al. (2007) Functional properties of neurons derived from in vitro reprogrammed postnatal astroglia. The Journal of neuroscience : the official journal of the Society for Neuroscience 27, 8654-8664

4 Heinrich, C. et al. (2010) Directing Astroglia from the Cerebral Cortex into Subtype Specific Functional Neurons. PLoS biology 8, e1000373

5 Heinrich, C. et al. (2011) Generation of subtype-specific neurons from postnatal astroglia of the mouse cerebral cortex. Nat Protoc 6, 214-228 
6 Karow, M. et al. (2012) Reprogramming of pericyte-derived cells of the adult human brain into induced neuronal cells. Cell stem cell 11, 471-476

7 Masserdotti, G. et al. (2015) Transcriptional Mechanisms of Proneural Factors and REST in Regulating Neuronal Reprogramming of Astrocytes. Cell stem cell 17, 74-88

8 Gascón, S. et al. (2016) Identification and Successful Negotiation of a Metabolic Checkpoint in Direct Neuronal Reprogramming. Cell stem cell 18, 396-409

9 Rivetti di Val Cervo, P. et al. (2017) Induction of functional dopamine neurons from human astrocytes in vitro and mouse astrocytes in a Parkinson's disease model. Nature Biotechnology 35, 444-452

10 Karow, M. et al. (2018) Direct pericyte-to-neuron reprogramming via unfolding of a neural stem cell-like program. Nature Neuroscience DOI: 10.1038/s41593-018-0168-3

11 Vierbuchen, T. and Wernig, M. (2011) Direct lineage conversions: unnatural but useful? Nature Biotechnology 29, 892-907

12 Yang, N. et al. (2011) Induced neuronal cells: how to make and define a neuron. Cell stem cell 9, 517-525

13 Wapinski, O.L. et al. (2013) Hierarchical Mechanisms for Direct Reprogramming of Fibroblasts to Neurons. Cell 155, 621-635

14 Smith, D.K. et al. (2016) Small Molecules Modulate Chromatin Accessibility to Promote NEUROG2-Mediated Fibroblast-to-Neuron Reprogramming. Stem Cell Reports 7, 955-969

15 Zhang, L. et al. (2015) Small Molecules Efficiently Reprogram Human Astroglial Cells into Functional Neurons. Cell stem cell 17, 735-747

16 Buffo, A. et al. (2005) Expression pattern of the transcription factor Olig2 in response to brain injuries: implications for neuronal repair. Proceedings of the National Academy of Sciences of the United States of America 102, 18183-18188

17 Ohori, Y. et al. (2006) Growth factor treatment and genetic manipulation stimulate neurogenesis and oligodendrogenesis by endogenous neural progenitors in the injured adult spinal cord. The Journal of neuroscience : the official journal of the Society for Neuroscience $26,11948-11960$

18 Torper, O. et al. (2013) Generation of induced neurons via direct conversion in vivo. 
Proceedings of the National Academy of Sciences of the United States of America 110, 70387043

19 Grande, A. et al. (2013) Environmental impact on direct neuronalreprogramming in vivo in the adult brain. Nature communications 4, 1-12

20 Niu, W. et al. (2013) In vivo reprogramming of astrocytes to neuroblasts in the adult brain. Nature Cell Biology 15, 1164-1175

21 Heinrich, C. et al. (2014) Sox2-mediated conversion of NG2 glia into induced neurons in the injured adult cerebral cortex. Stem Cell Reports 3, 1000-1014

22 Guo, Z. et al. (2014) In Vivo direct reprogramming of reactive glial cells into functional neurons after brain injury and in an Alzheimer's disease model. Cell stem cell 14, 188-202

23 Niu, W. et al. (2015) SOX2 reprograms resident astrocytes into neural progenitors in the adult brain. Stem Cell Reports 4, 780-794

24 Torper, O. et al. (2015) In Vivo Reprogramming of Striatal NG2 Glia into Functional Neurons that Integrate into Local Host Circuitry. CellReports 12, 474-481

25 Pereira, M. et al. (2017) Direct Reprogramming of Resident NG2 Glia into Neurons with Properties of Fast-Spiking Parvalbumin-Containing Interneurons. Stem Cell Reports 9, 742751

26 Treutlein, B. et al. (2016) Dissecting direct reprogramming from fibroblast to neuron using single-cell RNA-seq. Nature 534, 391-395

27 Tsunemoto, R. et al. (2018) Diverse reprogramming codes for neuronal identity. Nature 557, $375-380$

28 Matsuda, T. et al. (2019) Pioneer Factor NeuroD1 Rearranges Transcriptional and Epigenetic Profiles to Execute Microglia-Neuron Conversion. Neuron 101, 472-485.e7

29 Dimou, L. and Gotz, M. (2014) Glial Cells as Progenitors and Stem Cells: New Roles in the Healthy and Diseased Brain. Physiological reviews 94, 709-737

30 Robel, S. et al. (2011) The stem cell potential of glia: lessons from reactive gliosis. Nat. Rev. Neurosci. 12, 88-104

31 Sirko, S. et al. (2013) Reactive glia in the injured brain acquire stem cell properties in response to sonic hedgehog. [corrected]. Cell stem cell 12, 426-439 
32 Blum, R. et al. (2011) Neuronal network formation from reprogrammed early postnatal rat cortical glial cells. Cereb. Cortex 21, 413-424

33 Addis, R.C. et al. (2011) Efficient conversion of astrocytes to functional midbrain dopaminergic neurons using a single polycistronic vector. PloS one 6, e28719

34 Hughes, E.G. et al. (2013) Oligodendrocyte progenitors balance growth with self-repulsion to achieve homeostasis in the adult brain. Nature neuroscience 16, 668-676

35 Graham, V. et al. (2003) SOX2 functions to maintain neural progenitor identity. Neuron 39, 749-765

36 Rouaux, C. and Arlotta, P. (2013) Direct lineage reprogramming of post-mitotic callosal neurons into corticofugal neurons in vivo. Nature Cell Biology 15, 214-221

37 Rouaux, C. and Arlotta, P. (2010) Fezf2 directs the differentiation of corticofugal neurons from striatal progenitors in vivo. Nature neuroscience 13, 1345-1347

38 Chouchane, M. et al. (2017) Lineage Reprogramming of Astroglial Cells from Different Origins into Distinct Neuronal Subtypes. Stem Cell Reports 9, 162-176

39 Mosteiro, L. et al. (2016) Tissue damage and senescence provide critical signals for cellular reprogramming in vivo. Science 354 , aaf4445

40 Magnusson, J.P. et al. (2014) A latent neurogenic program in astrocytes regulated by Notch signaling in the mouse. Science 346, 237-241

41 Nato, G. et al. (2015) Striatal astrocytes produce neuroblasts in an excitotoxic model of Huntington's disease. Development 142, 840-845

42 Yin, J.-C. et al. (2019) Chemical Conversion of Human Fetal Astrocytes into Neurons through Modulation of Multiple Signaling Pathways. Stem Cell Reports 12, 488-501

43 Miyoshi, G. et al. (2007) Physiologically Distinct Temporal Cohorts of Cortical Interneurons Arise from Telencephalic Olig2-Expressing Precursors. The Journal of neuroscience : the official journal of the Society for Neuroscience 27, 7786-7798

$44 \mathrm{Hu}, \mathrm{X}$. et al. (2019) Region-Restrict Astrocytes Exhibit Heterogeneous Susceptibility to Neuronal Reprogramming. Stem Cell Reports 12, 290-304

45 Ruiz, S. et al. (2011) A high proliferation rate is required for cell reprogramming and maintenance of human embryonic stem cell identity. Curr. Biol. 21, 45-52 
46 Jørgensen, H.F. et al. (2009) REST selectively represses a subset of RE1-containing neuronal genes in mouse embryonic stem cells. Development 136, 715-721

47 Morris, S.A. (2016) Direct lineage reprogramming via pioneer factors; a detour through developmental gene regulatory networks. Development 143, 2696-2705

48 Takahashi, K. and Yamanaka, S. (2006) Induction of pluripotent stem cells from mouse embryonic and adult fibroblast cultures by defined factors. Cell 126, 663-676

49 Bertolini, J.A. et al. (2019) Mapping the Global Chromatin Connectivity Network for Sox2 Function in Neural Stem Cell Maintenance. Cell stem cell 24, 462-476.e6

50 Tsacopoulos, M. and Magistretti, P.J. (1996) Metabolic coupling between glia and neurons. The Journal of neuroscience : the official journal of the Society for Neuroscience 16, 877-885

51 Schimmel, S. et al. (2017) Neuroinflammation in traumatic brain injury: A chronic response to an acute injury. Brain Circ 3, 135

$52 \mathrm{Su}, \mathrm{Z}$. et al. (2014) In vivo conversion of astrocytes to neurons in the injured adult spinal cord. Nature communications 5, 1-15

53 Chanda, S. et al. (2019) Direct Reprogramming of Human Neurons Identifies MARCKSL1 as a Pathogenic Mediator of Valproic Acid-Induced Teratogenicity. Cell stem cell DOI: 10.1016/j.stem.2019.04.021

54 la Rossa, De, A. et al. (2013) In vivo reprogramming of circuit connectivity in postmitotic neocortical neurons. Nature Neuroscience 16, 193-200

55 Niu, W. et al. (2018) Phenotypic Reprogramming of Striatal Neurons into Dopaminergic Neuron-like Cells in the Adult Mouse Brain. Stem Cell Reports 11, 1156-1170

56 Amamoto, R. and Arlotta, P. (2014) Development-Inspired Reprogramming of the Mammalian Central Nervous System. Science 343, 1239882-1239882

57 Foust, K.D. et al. (2009) Intravascular AAV9 preferentially targets neonatal neurons and adult astrocytes. Nature biotechnology 27, 59-65

58 Brulet, R. et al. (2017) NEUROD1 Instructs Neuronal Conversion in Non-Reactive Astrocytes. Stem Cell Reports 8, 1506-1515

59 Wang, Y. et al. (2013) Systemic delivery of modified mRNA encoding herpes simplex virus 1 thymidine kinase for targeted cancer gene therapy. Mol Ther 21, 358-367 
60 Ridder, K. et al. (2014) Extracellular vesicle-mediated transfer of genetic information between the hematopoietic system and the brain in response to inflammation. PLoS biology 12, e1001874

61 Yoo, J. et al. (2017) Electromagnetized gold nanoparticles mediate direct lineage reprogramming into induced dopamine neurons in vivo for Parkinson's disease therapy. Nature Nanotechnology 12, 1006-1014

62 Cheng, L. et al. (2015) Direct conversion of astrocytes into neuronal cells by drug cocktail. Cell research 25, 1269-1272

\section{Glossary}

Activin/nodal: Members of the TGF $\beta$ superfamily of proteins that influence self-renewal, differentiation and proliferation in neural stem cells.

Chromatin remodeling: Chromatin remodeling is the rearrangement of chromatin architecture (histones and DNA) that regulates the dynamic access to the packaged DNA. Chromatin remodeling is highly implicated in epigenetics. Epigenetic modifications to histone proteins such as methylation/demethylation and acetylation/deacetylation alter the structure of chromatin resulting in transcriptional activation or repression.

Fate determinant: Genes with specific temporal and spatial expression during development that instruct the attainment of specific cell types/phenotypes.

GAD65/67: Glutamic Acid Decarboxylase 65/67 are two isoforms of the enzyme responsible for production of GABA.

miRNA: microRNA are short non-coding sequences of RNA that can bind to mRNA to inhibit translation.

NG2 glia: Glial cells widely dispersed through the adult CNS parenchyma, also known as oligodendrocyte progenitor cells (OPCs). Their functions are not fully known yet.

Notch signaling: A highly conserved signaling pathway that maintains undifferentiated state of neural stem cells.

NSCs: Neural Stem Cells are self-renewing multipotent cells that give rise to neurons and glia. 
Oxidative stress: A detrimental, imbalanced state arising from an excess of reactive oxygen species (ROS) production during metabolism.

Reactive gliosis: A process in which glia residing in the parenchyma proliferate and respond to injury in the CNS.

REST: RE1-Silencing Transcription factor is a repressor of transcription, capable of silencing multiple genes at the site of histones and DNA.

TGF $\beta$ : A superfamily of signaling proteins that affect cell morphogenesis, differentiation and survival.

vGluT1: Vesicular Glutamate Transporter 1 is a marker for glutamatergic neurons.

\section{Box1. Chemical reprogramming of human astrocytes into neurons}

To date, several studies have converted human astrocytes into hiNs using small molecule cocktails (Figure 2E). Glia-to-neuron conversion exclusively by small molecules could represent an attractive approach circumventing the need for virally-driven expression of TFs and invasive surgery to reach the targeted region for future clinical applications. Chen and colleagues identified a combination of nine small molecules capable of reprogramming human fetal astrocytes into hiNs in vitro but not mouse astrocytes [15]. The selected compounds functioned to inhibit glial signaling (SB431542, LDN193189) and activate neuronal differentiation pathways (TTNPB, CHIR99021, DAPT, SAG, purmorphamine), promote survival (thiazovivin), and increase chromatin plasticity (VPA), and were replaced subsequently by neurotrophic factors (BDNF, NT3, IGF1). Sequential treatment with these molecules over ten days produced MAP2/NeuN-positive hiNs, capable of long-term survival in culture and functional maturation, as evidenced with patch clamp recoding and $\mathrm{Ca}^{2+}$ imaging. hiNs were also capable of network formation in vitro and exhibited properties of forebrain glutamatergic neurons. Chemical compounds were shown to induce epigenetic regulations and endogenous expression of Ascl1, Neurog2 and NeuroD1. In a later study, however, the authors observed that application of four of the above molecules simultaneously (SB43152, LDN193189, CHIR99021 and DAPT) to inhibit respectively TGF- $\beta$, BMP, GSK-3 $\beta$, and Notch signaling was sufficient to generate functional hiNs from human fetal astrocytes and at greater efficiencies than the nine compound cocktail [42]. Interestingly, during chemical 
reprogramming, REST was downregulated whereas MECP2 expression increased significantly. hiNs adopted varying phenotypes based on astrocyte origin, with cortical astrocytes producing glutamatergic neurons, and midbrain astrocytes producing glutamatergic and GABAergic neurons, albeit at lower rates, further illustrating that intrinsic factors expressed in distinct astrocyte subpopulations influence cell-fate conversion. However, despite several attempts, the authors failed in inducing astrocyte conversion in the mouse brain in vivo using the selected chemical cocktail $[15,42]$. One apparent hurdle is how to maintain a constant concentration of small molecules within the brain to induce astrocyte-to-neuron conversion [42]. Importantly, a major challenge will be to prevent off-target reprogramming upon systemic administration, and to determine to which extent brain-resident cells not targeted to be reprogrammed, will be affected in their intrinsic identity and functions upon prolonged exposure to chemical compounds. Valproic acid, a teratogenic drug, was recently showed to alter properties of developing neurons [53].

\section{Box2. Endogenous neurons: A source cell type for induced neurons}

Seminal studies showed that neurons themselves can be turned from one class into alternate neuronal subtypes within the brain $[36,37,54,55]$. Neuron-to-neuron conversion could be an alternative strategy to regenerate lost neuronal subtypes offering attractive features. Converting neighboring neurons naturally resistant to the same pathology and sharing similar neurotransmitter identity, ability to send long-distance axons, or location into a circuit may facilitate the generation of highly-specialized neurons, ease their network integration, and possibly reduce off-target connectivity [56]. The Arlotta lab showed that overexpressing Fezf2 in neural progenitors of the lateral ganglionic eminence in mouse embryos, which would normally give rise to GABAergic neurons of the striatum and olfactory bulb, caused a complete fate-switch to generate glutamatergic neurons exhibiting molecular properties of corticofugal projection neurons of the cerebral cortex within the striatum (CFuPNs) [37]. Several neurons acquired morphology and connectivity similar to CFuPNs including axonal projections to the thalamus and cerebral peduncle corresponding to CFuPN appropriate targets. This fate-switch occurred despite ectopic location within the striatum, thus demonstrating that cell-autonomous signaling driving neuron type-specific development can override extracellular niche restrictions outside the cortex [37]. Furthermore, 
conversion between neuronal subtypes was not restricted to embryonic development as Fezf2 expression induced in vivo reprogramming of postmitotic early postnatal callosal projection neurons of layer-II/III into layer-V/VI CFuPNs, as revealed by acquisition of new molecular identity and axonal connectivity directed below the cortex [36]. Neuronal conversion was nonetheless reliant on some degree of nuclear plasticity during a critical period of the postmitotic life of a neuron before progressive fate-restriction and loss of reprogramming ability [36]. Similarly, Fezf2 could also instruct layer-IV postmitotic spiny neurons to acquire molecular features of layer-Vb neurons, acquiring their specific pyramidal morphology, intracortical inputs, axonal projections into subcortical structures, and electrophysiological signature [54].

While postmitotic mechanisms restricting conversion between neuronal fates likely become more active when neurons age, a maintained capacity for in vivo neuron-to-neuron phenotype reprogramming was recently described in the adult mouse striatum [55]. Strikingly, striatal medium spiny neurons were converted into dopaminergic-like iNs by expression of Sox2, Nurr1, FoxA2, Lmx1A combined with chromatin remodeling. iNs expressed stereotypical markers and exhibited electrophysiological properties analogous to endogenous dopaminergic neurons [55]. Of note however, remnants of spiny neuron identity were still detectable in iNs, suggesting that complete silencing of source cell identities seems not to be a prerequisite for successful neuron-to-neuron transition [55].

\section{Clinician's Corner}

- Acute CNS injury and chronic neurodegenerative diseases are associated with irreversible loss of neurons and permanent functional deficits. Direct lineage reprogramming, aiming at converting brain-resident, non-neuronal cells into clinically-relevant iNs, has emerged as a promising cell-replacement strategy in regenerative medicine. Reactive glia at the injury site revealed to be valuable source cell types for neuronal reprogramming. Forced expression of TFs and/or exposure to small molecules successfully instructed murine glial cells in vitro and in the injured brain in vivo to generate glutamatergic, GABAergic or dopaminergic iNs, exhibiting some degree of integration within endogenous networks. Importantly, human astrocytes and pericytes were also converted into iNs entering distinct neuronal lineages. 
- Long-term stability of iNs appear critical for long-lasting effects on networks and functions. While several studies suggested maintenance of the reprogrammed status of iNs, iN stability over time in absence of reversal to their original cellular identity needs to be confirmed before clinical translation.

- Another important issue is the ability to drive efficient generation of iNs harboring the desired phenotype to obtain an adequate therapeutic potency. Most studies reported high efficiencies of neuronal conversion including in the injured brain. With respect to an ongoing quest for highest conversion efficiencies, a potential flip-side of the coin could be a partial depletion of the source cell types converted into iNs. Thus, undesired detrimental effects related to the loss of glia or pericytes need to be carefully evaluated. Targeting a source population endowed with capacity for proliferation and homeostatic control of density could be preferable to avoid potential drawbacks.

Regarding clinical translation, reprogramming gene delivery methods have to be safe, efficient and specific to targeted cell types. Being non-integrative, adeno-associated virus (AAV) is an attractive viral delivery system. One problem may be targeting the desired area, which may still require local surgical interventions. Recently, however, AAV9 crossing the blood-brain barrier was used to induce striatal astrocyte reprogramming through an intravascular route $[57,58]$. Alternatively, non-invasive systemic delivery of modified RNAs [59] or small molecules (Box1), extracellular microvesicles [60], or electromagnetized gold nanoparticles [61] can be envisaged.

- Translational research needs to be conducted in appropriate disease models to reveal the potential of direct lineage reprogramming as a viable therapeutic approach for CNS repair. Although there is still a long way to go before moving from bench to bedside, lineage reprogramming could open doors to alternative and effective therapeutic approaches, allowing use of patient-specific cells towards brain repair.

Table 1: Direct lineage reprogramming of murine and human brain-resident cells into induced neurons. 


\begin{tabular}{|c|c|c|c|c|c|c|c|c|}
\hline \multicolumn{9}{|c|}{ Direct Reprogramming of Murine Cells } \\
\hline & Sour & e cell & Lesion & Transcription & Small Molecules & $\begin{array}{l}\text { iN Phenotype } \\
\end{array}$ & Functional outcome / Electrophysiology & Ref \\
\hline & in vitro & in vivo & Model & Factor(s) & & & & \\
\hline \multirow{4}{*}{ 希 } & & $\begin{array}{c}\text { Reactive } \\
\text { glia }\end{array}$ & SWI & Olig2VP16; Pax6 & & DCX, PAX6 & & [16] \\
\hline & & $\begin{array}{l}\text { Reactive } \\
\text { glia }\end{array}$ & $\begin{array}{l}\mathrm{SWI} / \\
\mathrm{ISCH}\end{array}$ & Neurog2 & EGF, FGF2 & $\begin{array}{l}\text { Glutamatergic - NEUN, GLU, } \\
\text { GLUR2/3, BHLHB5 }\end{array}$ & & [19] \\
\hline & & $\begin{array}{l}\text { Reactive } \\
\text { glia }\end{array}$ & SWI & Pax6 & EGF, FGF2 & $D C X$ & & [19] \\
\hline & & $\begin{array}{l}\text { Reactive } \\
\text { glia }\end{array}$ & SWI & Neurog $2+B c 12$ & $\begin{array}{l}\text { calcitriol, } \alpha- \\
\text { Tocotrienol }\end{array}$ & $\begin{array}{l}\text { Glutamatergic - NEUN, CTIP2, } \\
\text { FOXP2 }\end{array}$ & & [8] \\
\hline \multirow{2}{*}{ 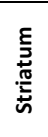 } & & $\begin{array}{l}\text { Reactive } \\
\text { glia }\end{array}$ & SWI & Pax6 & EGF, FGF2 & DCX & & [19] \\
\hline & & $\begin{array}{l}\text { Reactive } \\
\text { glia }\end{array}$ & SWI & Neurog2 & EGF, FGF2 & GABAergic - NEUN, GABA, ISL1 & & [19] \\
\hline$\stackrel{ن}{\dot{n}}$ & & $\begin{array}{c}\text { Reactive } \\
\text { glia }\end{array}$ & SCl & Neurog2 & EGF, FGF2, BDNF & HuC/D, TUJ1, MAP2 & & [17] \\
\hline \multirow{18}{*}{ 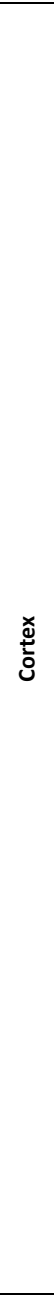 } & astrocytes & & & Neurog2 & & TUJ1, TBR1 & $\begin{array}{l}\text { Repetitive discharges of APs; spontaneous synaptic } \\
\text { inputs. }\end{array}$ & [3] \\
\hline & astrocytes & & & Ascl1 & & TUJ1 & Repetitive discharges of APs. & [3] \\
\hline & astrocytes & & & Pax6 & & TUJ1 & $\begin{array}{l}\text { Repetitive discharges of APs; spontaneous synaptic } \\
\text { inputs. }\end{array}$ & {$[2,3]$} \\
\hline & astrocytes & & & Neurog2 & & $\begin{array}{l}\text { Glutamatergic - MAP2, } \\
\text { CAMKIlla, VGLUT1, TBR1, TBR2 }\end{array}$ & $\begin{array}{l}\text { Repetitive AP firing; spontaneous synaptic inputs; } \\
\text { iNs establish functional glutamatergic synapses and } \\
\text { elicit CNQX-sensitive postsynaptic currents. }\end{array}$ & $\begin{array}{l}{[4]} \\
{[5]}\end{array}$ \\
\hline & astrocytes & & & $D \mid x 2$ & & $\begin{array}{c}\text { GABAergic - MAP2, GAD67, } \\
\text { CALR, VGAT }\end{array}$ & $\begin{array}{l}\text { AP firing with regular, stuttering and low-threshold } \\
\text { burst spiking (non-fast spiking interneurons); } \\
\text { spontaneous synaptic inputs; iNs establish } \\
\text { functional GABAergic synapses and elicit } \\
\text { bicuculline-sensitive postsynaptic currents. }\end{array}$ & $\begin{array}{l}{[4]} \\
{[5]}\end{array}$ \\
\hline & $\begin{array}{l}\text { astrocytes } \\
\text { (NG2) }\end{array}$ & & & Neurog2 & & Glutamatergic - VGLUT1 & $\begin{array}{l}\text { Synchronous Ca2+ oscillations, and AP firing; iNs } \\
\text { establish functional glutamatergic synapses and } \\
\text { elicit CNQX-sensitive postsynaptic currents. }\end{array}$ & [32] \\
\hline & astrocytes & & & $\begin{array}{l}\text { Ascl1 }+\operatorname{Lm} \times 1 b+ \\
\text { Nurr1 }\end{array}$ & & Dopaminergic - TH, GIRK2 & DA release (HPLC). & [33] \\
\hline & astrocytes & & & NeuroD1 & & $\begin{array}{c}\text { Glutamatergic - NEUN, VGLUT1, } \\
\text { CTIP2, OTX1, TBR1 }\end{array}$ & $\begin{array}{l}\text { Repetitive AP firing and CNQX-sensitive synaptic } \\
\text { events. }\end{array}$ & [22] \\
\hline & & astrocytes & $\begin{array}{l}\text { SWI } / 5 x \\
\text { FAD Tg }\end{array}$ & NeuroD1 & & TUJ1, NEUN & Synaptic events. & [22] \\
\hline & astrocytes & & & Neurog2; Ascl1 & & TUJ1 & & [7] \\
\hline & astrocytes & & & NeuroD4 & & Glutamatergic - TUJ1 & $\begin{array}{l}\text { Repetitive AP firing; spontaneous synaptic inputs; } \\
\text { glutamatergic synapses (NBQX-sensitive). }\end{array}$ & [7] \\
\hline & astrocytes & & & NeuroD4 + Insm1 & & Glutamatergic - TUJ1, VGLUT1 & $\begin{array}{l}\text { Repetitive AP firing; spontaneous synaptic inputs; } \\
\text { glutamatergic synapses (NBQX-sensitive). }\end{array}$ & [7] \\
\hline & astrocytes & & & NeuroD4 + Prox1 & & TUJ1 & Repetitive AP firing. & [7] \\
\hline & astrocytes & & & & $\begin{array}{l}\text { VPA, Chir99021, } \\
\text { Respox }\end{array}$ & NEUN & $\begin{array}{l}\text { Repetitive AP firing and spontaneous synaptic } \\
\text { inputs. }\end{array}$ & [62] \\
\hline & astrocytes & & & Ascl1 + Bcl2 & $\begin{array}{l}\text { Z-VAD-FMK; } \\
\text { Necrostatin-1, } \\
\text { liproxstatin-1, } \alpha \text { - } \\
\text { Tocopherol, Fk }\end{array}$ & MAP2, NEUN & AP firing & [8] \\
\hline & astrocytes & & & Neurog $2+B c 12$ & & $\begin{array}{c}\text { Glutamatergic - MAP2, NEUN, } \\
\text { VGLUT1 }\end{array}$ & & [8] \\
\hline & astrocytes & $\begin{array}{l}\text { Graft: } \\
\text { SVZ }\end{array}$ & & Ascl1 & & $\begin{array}{l}\text { Granule cell layer-like - NEUN } \\
\text { (Olfactory bulb neurons) }\end{array}$ & & [38] \\
\hline & & $\begin{array}{l}\text { Graft: } \mathrm{Cx} \\
\text { PN mouse }\end{array}$ & & Neurog2 & & NEUN, CUX1 & & \\
\hline \multirow{3}{*}{ 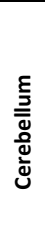 } & astrocytes & & & Ascl1 & & $\begin{array}{l}\text { Glutamatergic and GABAergic - } \\
\text { MAP2, NEUN, SYN1, GABA, GLU, } \\
\text { CALB, PV, CTIP2 }\end{array}$ & AP firing. & [38] \\
\hline & astrocytes & & & Neurog2 & & $\begin{array}{c}\text { Glutamatergic and GABAergic - } \\
\text { MAP2, NEUN, SYN1, GABA, GLU, } \\
\text { CALB, PV }\end{array}$ & AP firing. & [38] \\
\hline & astrocytes & $\begin{array}{l}\text { Graft: } \\
\text { SVZ }\end{array}$ & & Ascl1 & & $\begin{array}{l}\text { Periglomerular - NEUN } \\
\text { (Olfactory bulb neurons) }\end{array}$ & & [38] \\
\hline \multirow{4}{*}{ 密 } & & astrocytes & & $\begin{array}{l}\text { Ascl1 + Brn2a + } \\
\text { Myt11 }\end{array}$ & & NEUN & & [18] \\
\hline & & astrocytes & $\begin{array}{c}6- \\
\text { OHDA }\end{array}$ & $\begin{array}{l}\text { NeuroD1 + Ascl1 } \\
+ \text { Lm×1a+ miR218 }\end{array}$ & & $\begin{array}{l}\text { Dopaminergic - TH, NEUN, } \\
\text { SLC6A3, NR4A2, PBX1 }\end{array}$ & $\begin{array}{l}\text { Uptake/release of dopamine; AP firing and delayed } \\
\text { voltage rectification (biophysical signature of DA } \\
\text { neurons); Rescue of 6-OHDA-induced motor } \\
\text { deficits. }\end{array}$ & [9] \\
\hline & & astrocytes & & $\begin{array}{l}\text { Notch reduction } \\
\text { via } \mathrm{RBP}-\mathrm{Jk} \\
\text { deletion }\end{array}$ & & NEUN & & [40] \\
\hline & & astrocytes & & Sox2 & BNDF, Noggin; VPA & NEUN, CALR & AP firing and spontaneous synaptic inputs. & $\begin{array}{l}{[20,} \\
23]\end{array}$ \\
\hline
\end{tabular}




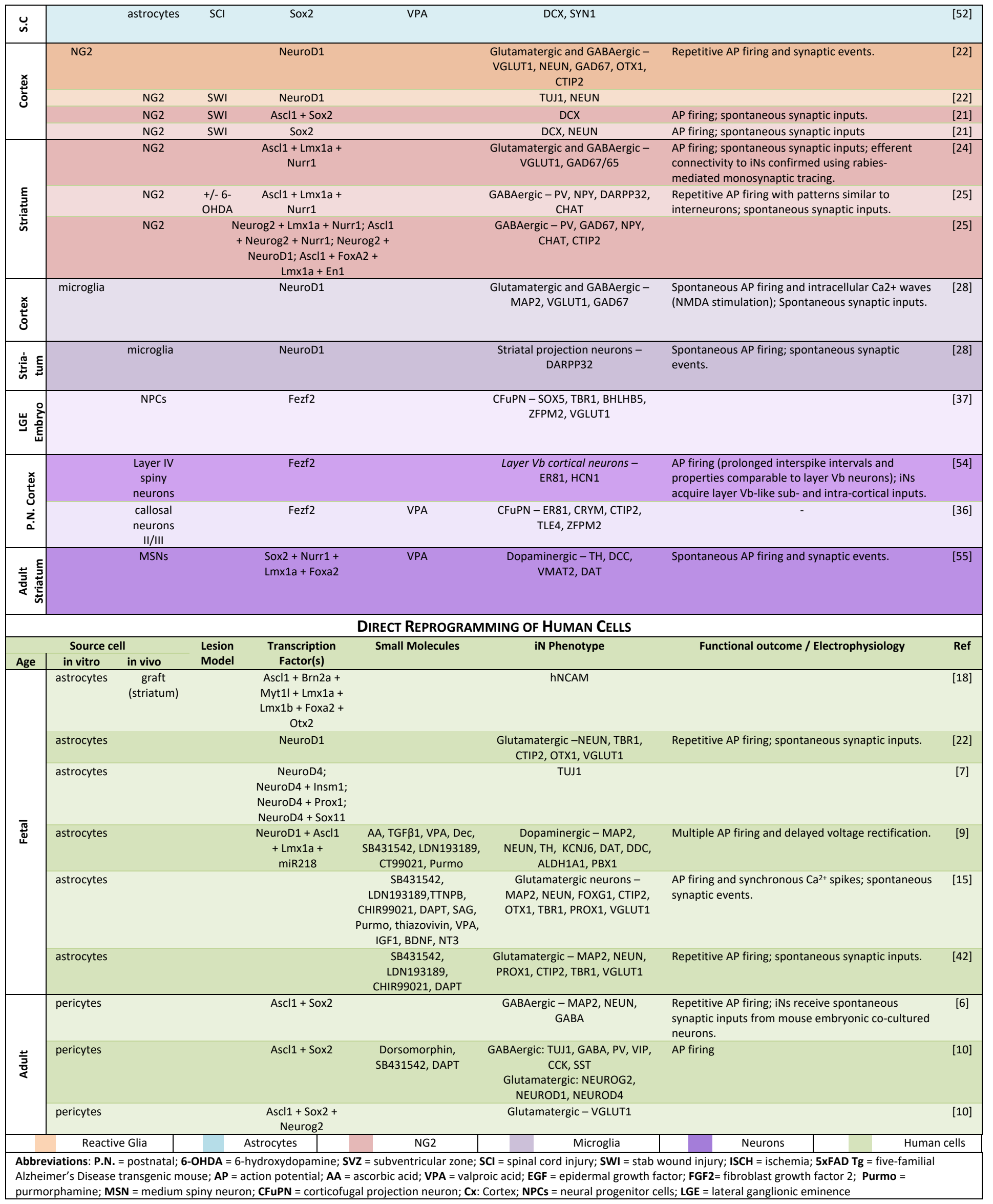




\section{Figure Legends}

\section{Figure 1: Direct lineage reprogramming of murine glial cells into iNs}

Studies are presented according to the cell-type involved (Proliferating glia (B,F), astrocytes: green $(A, E), N G 2$ glia: red $(D, H)$, microglia: blue $(C, G))$ and the brain region studied (cortex: upper blue panels, striatum: lower brown panels). Results obtained in vitro (Petri dish) and in vivo (mouse, bandage represents brain injury) are presented separately. Key transcription factors used are indicated in black. Treatment with small molecules is indicated in red and growth factors in green. a-Toc: $\alpha$-Tocopherol, BDNF: brain-derived neurotrophic factor, EGF: epidermal growth factor, FGF2: basic fibroblast growth factor, FK: Forskolin, iN: induced neuron, SWI: stab-wound injury, VitD: vitamin D, VitE: vitamin E, VPA: valproic acid.

\section{Figure 2: Direct lineage reprogramming of human brain-derived cells into iNs}

Several cell types of human origin are used for direct lineage reprogramming, including adult pericytes (purple, B) and fetal astrocytes (A, C, D, E) either immortalized (green) or in primary cultures (red). Key transcription factors and miRNA used are indicated in black. Treatment with small molecules is indicated in red and growth factors in green.

AP: action potential, iN: induced neuron, NSC: neural stem cell.

Figure 3 (Key figure): Multifactorial control of direct lineage reprogramming: Schematic view

TF-induced lineage reprogramming is affected by several factors (represented by gearwheels) that can either potentiate or be an obstacle to the neuronal conversion process. For each factor, some parameters known to affect reprogramming are highlighted (red and green arrows for parameters with negative and positive impact, respectively). 


\section{A Reprogramming cortical astrocytes}

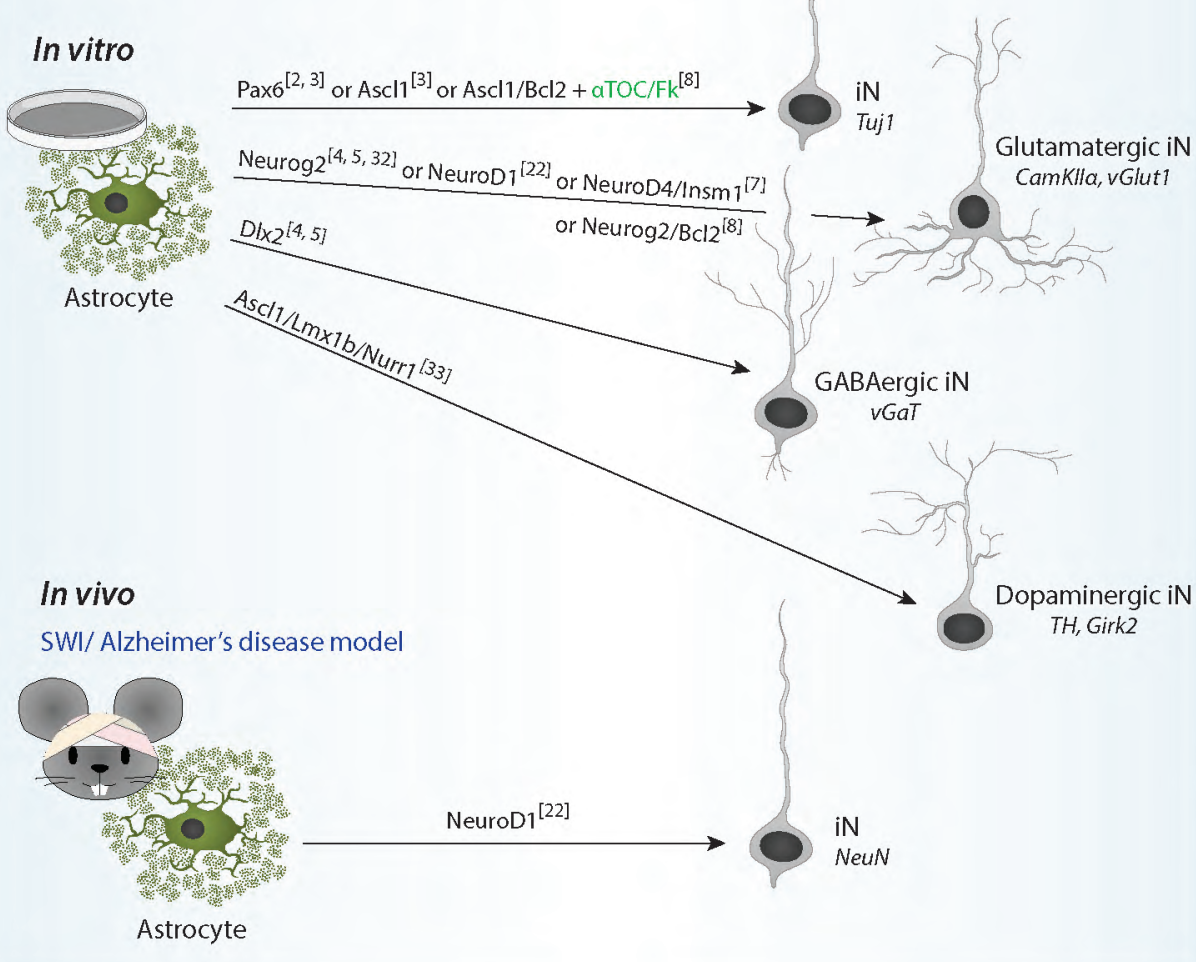

\section{Reprogramming cortical microglia}

In vitro

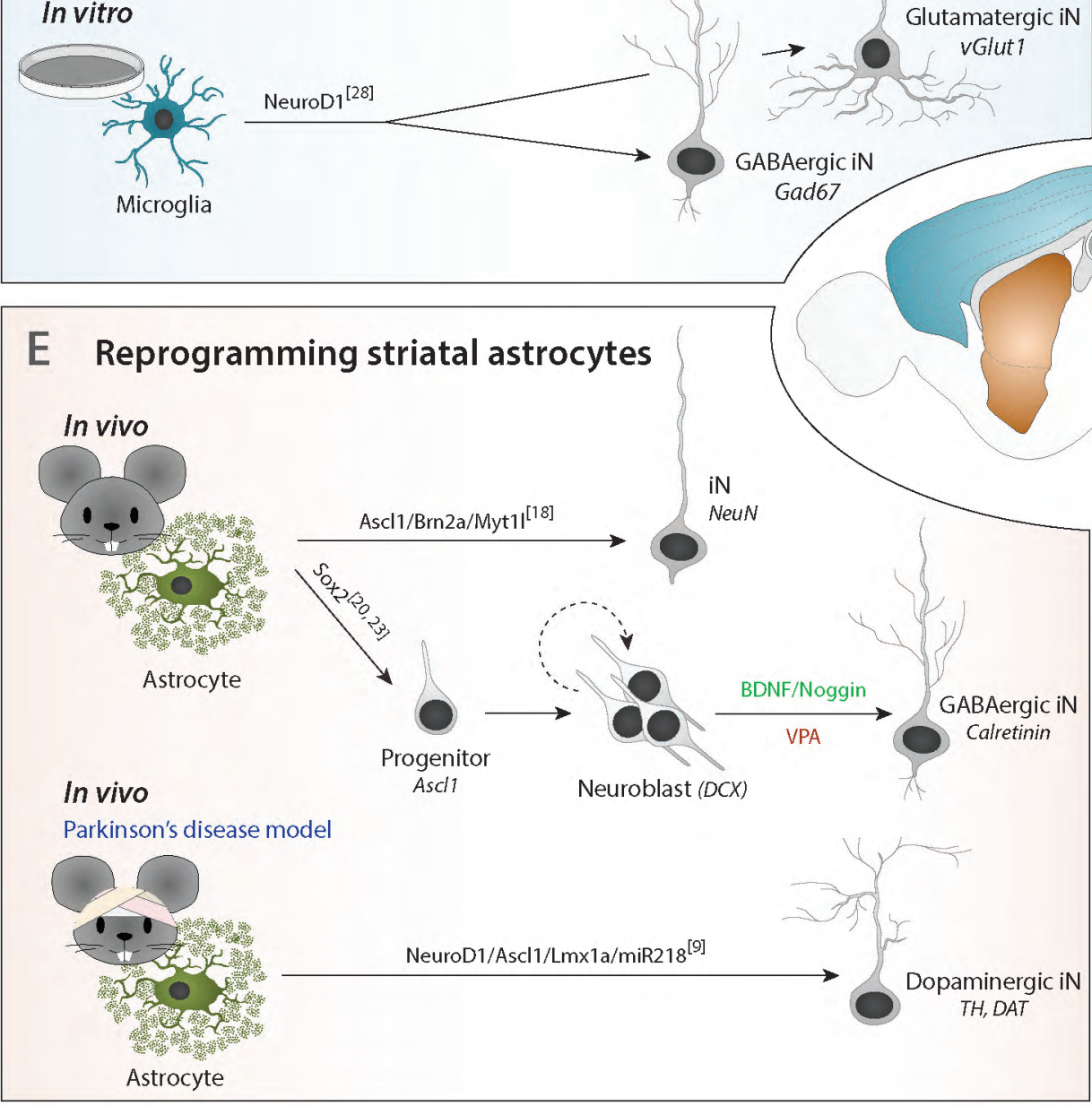

\section{G} In vivo

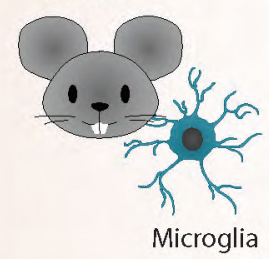

\section{B Reprogramming cortical proliferating glia}

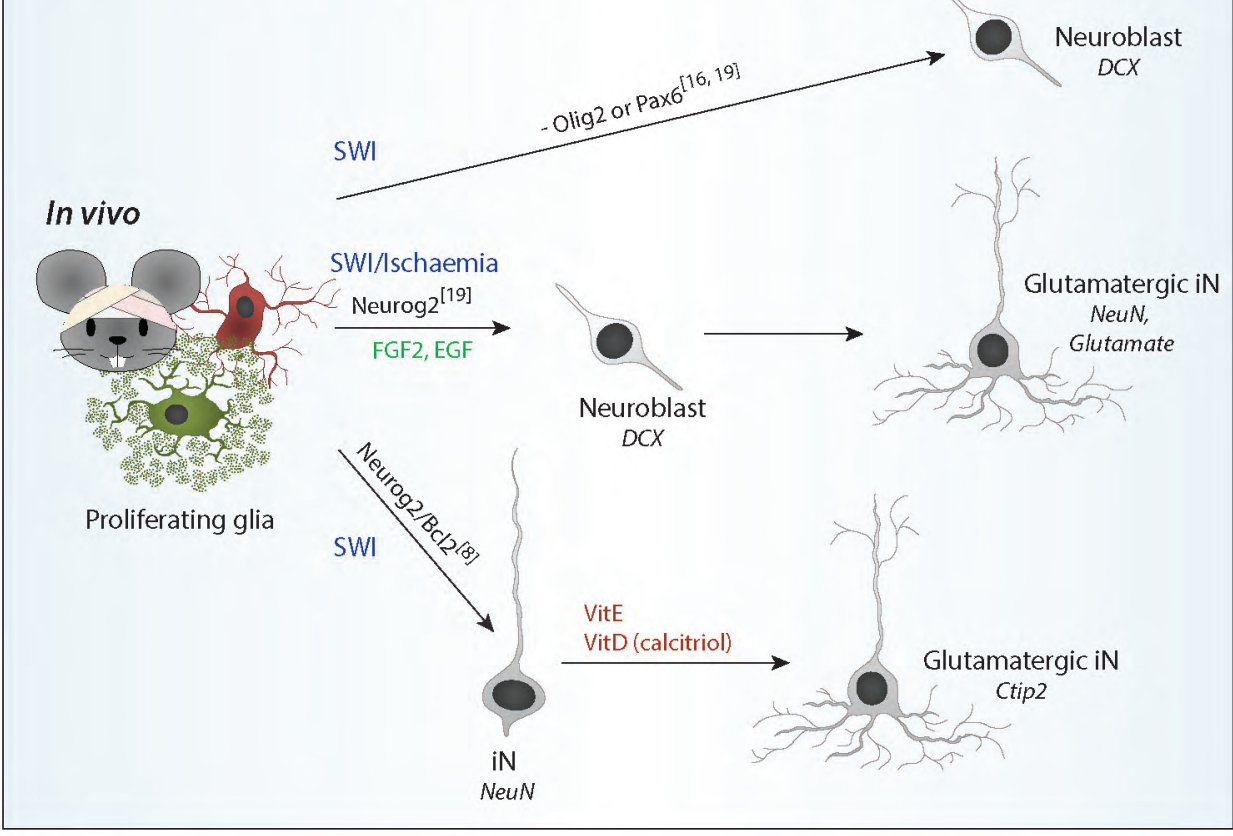

\section{Reprogramming cortical NG2 glia}

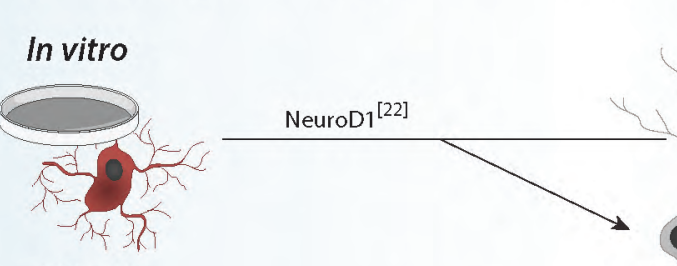

NG2 glia

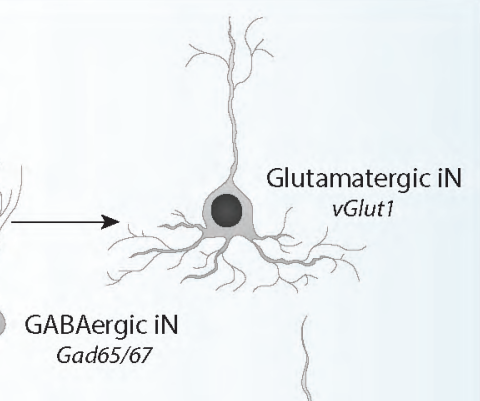

GABAergic iN
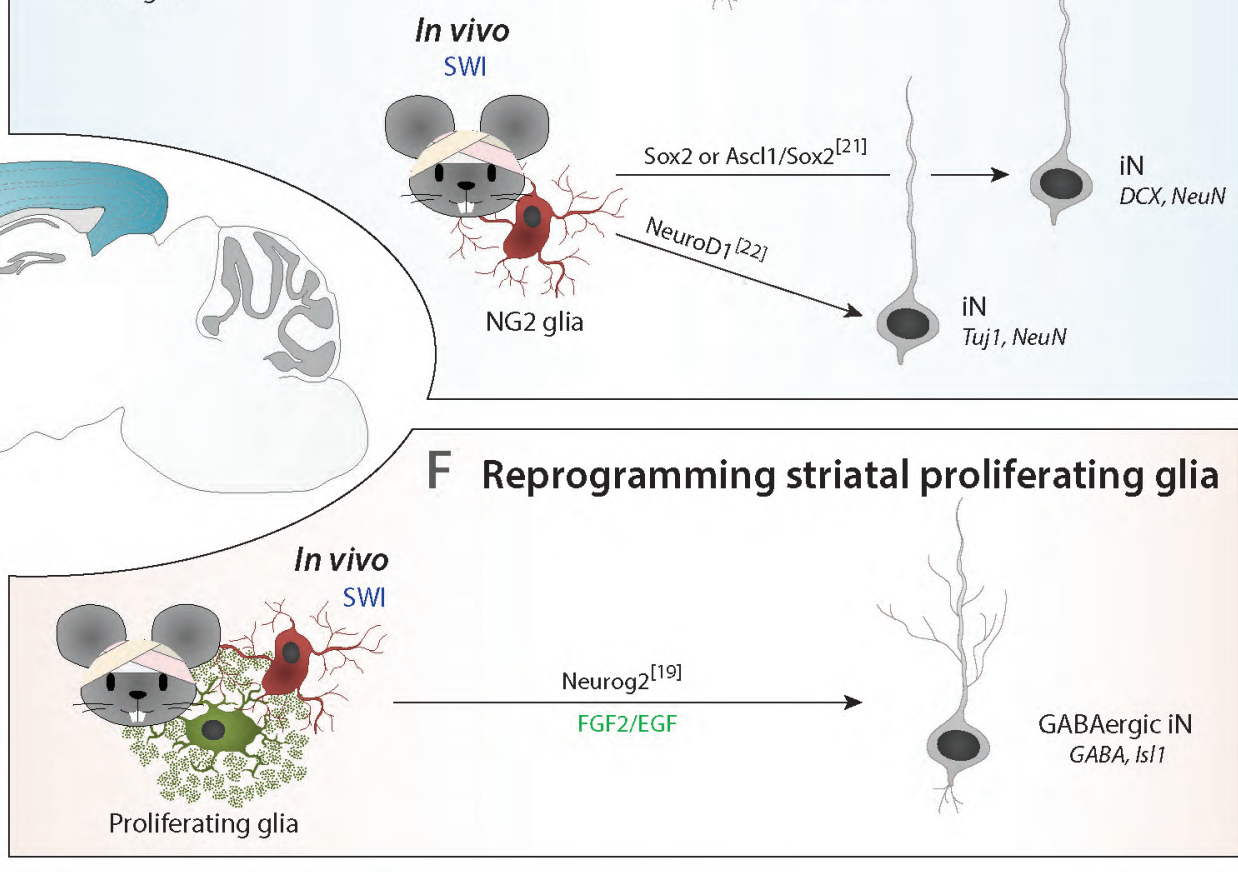

\section{H Reprogramming striatal NG2 glia}

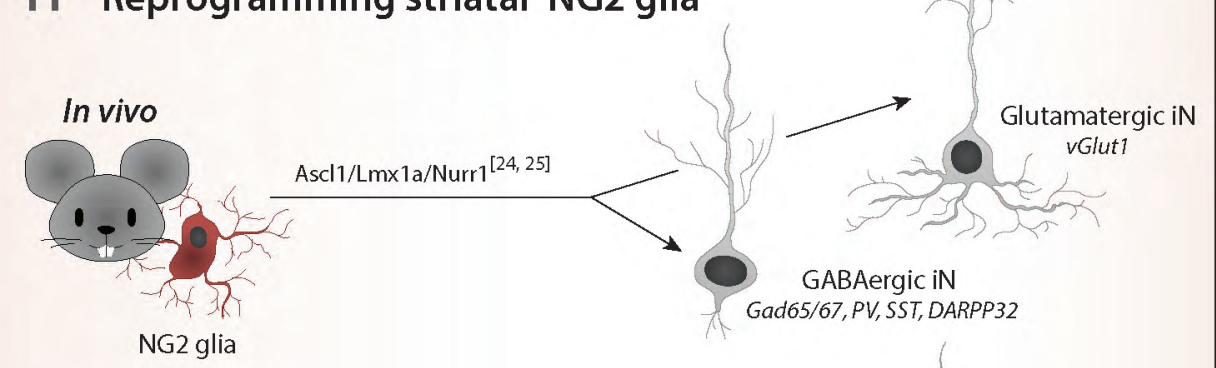

\section{In vivo}

Parkinson's disease model

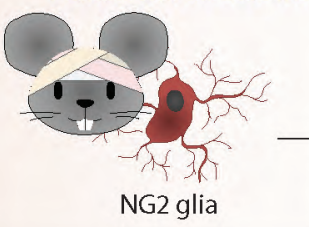


FIGURE 2

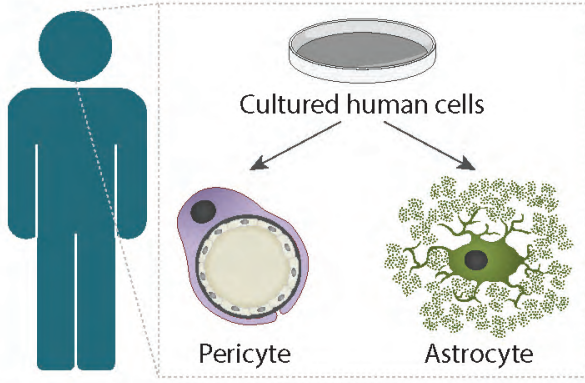

B Reprogramming human adult pericyte
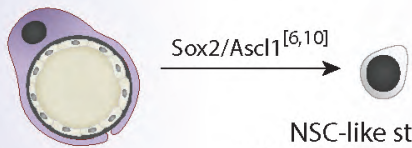

NSC-like state

Competent

Pericyte

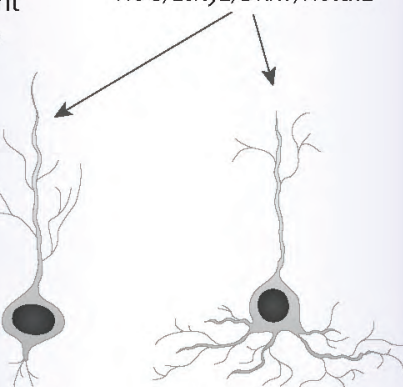

synaptic input

GABAergic iN GABA, VIP, CCK, SST

Glutamatergic iN

Neurog2, NeuroD1, NeuroD4

\section{Reprogramming human fetal astrocytes} In vitro conversion

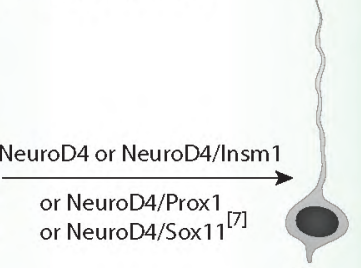

iN

Astrocyte

Blil-tubulin

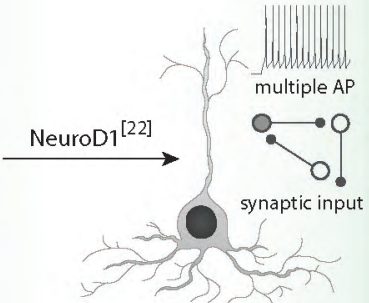

Glutamatergic iN vGlut1, Trb1, Ctip2, Otx1

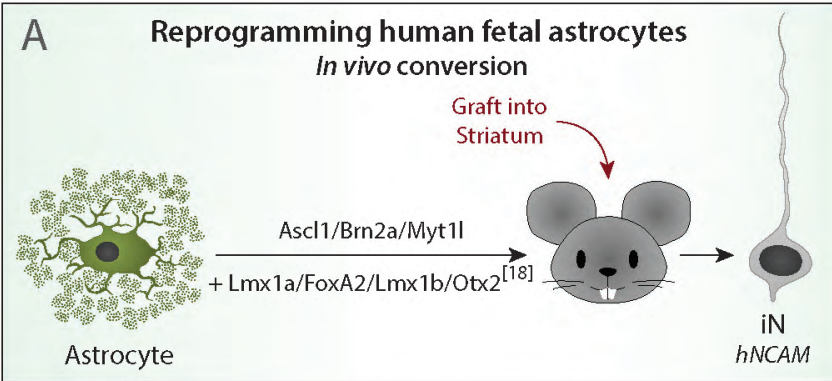

C Reprogramming human fetal astrocytes Promoting neuronal subtype identity

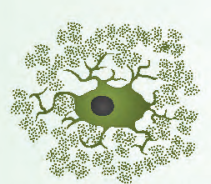

Immortalized

Astrocyte
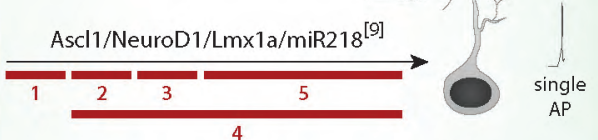

Dopaminergic iN

TH, DAT, DDC, VMAT2, ALDH1A1, KCNJ6

\begin{tabular}{|llrl}
\hline 1 & TGF $\beta 1$ & 2 & Chromatin remodeling agents \\
3 & Dual-Smad inhibitors & 4 & Chromatin remodeling factor \\
5 & Midbrain patterning signals, GSK3 $\beta$ inhibition
\end{tabular}

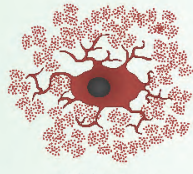

Primary

Astrocyte

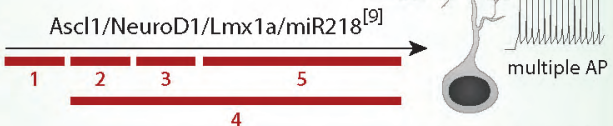

Dopaminergic iN TH, DAT, DDC, ALDHIA1, KCNJ6, PBX1

\section{E Reprogramming human fetal astrocytes Small molecule-induced reprogramming}
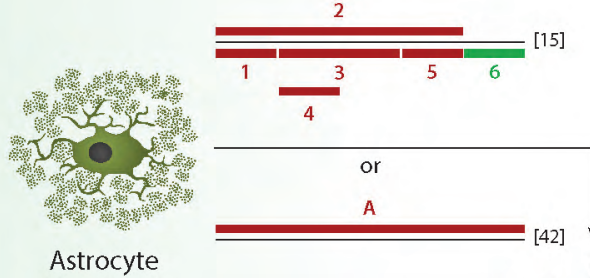

Astrocyte

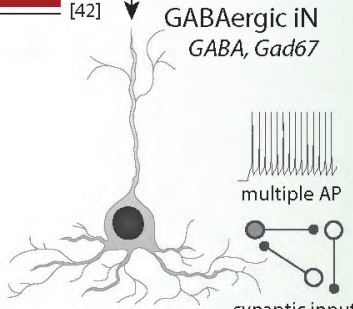

Glutamatergic iN

A Inhibitors of TGF $\beta / B M M_{\text {I }}$ GSK3 $\beta$ and Notch signalling 


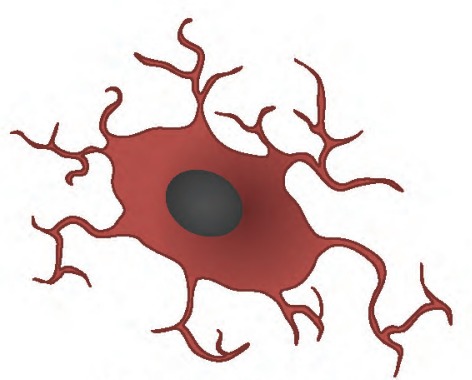

Brain resident cell

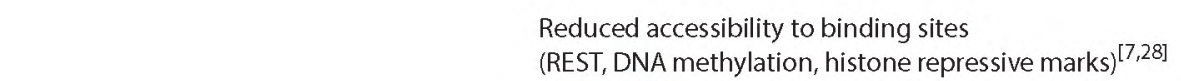

Accessibility to binding sites histone active marks) ${ }^{[2]}$

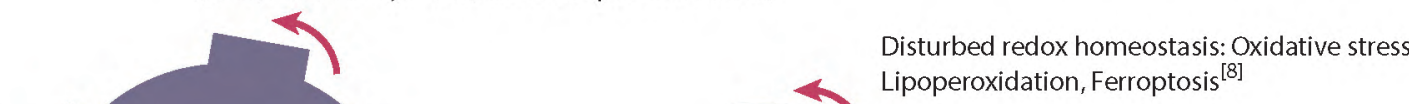

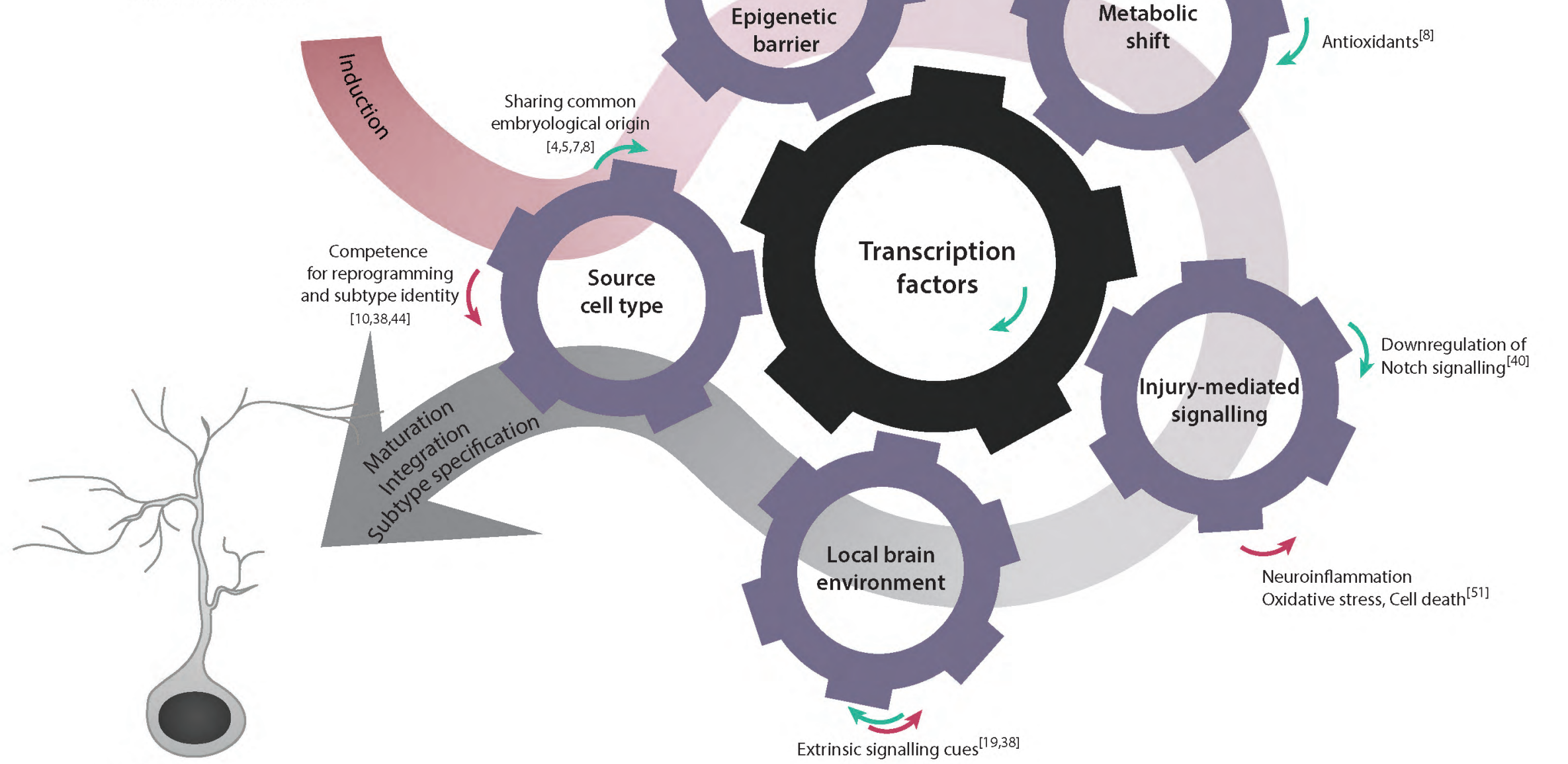

